\title{
Original Ofrticle \\ Adrenal histoplasmosis diagnosed by endoscopic ultrasound guided fine needle aspiration: experience of 10 patients
}

\author{
Rajiv Ranjan Singh ${ }^{1}$, Narendra S Choudhary ${ }^{1}$, Rajesh \\ Puri $^{1}$, Mridula Guleria ${ }^{2}$, Haimanti Sarin ${ }^{2}$, Mukesh Nasa ${ }^{1}$, \\ Rinkesh Bansal $^{1}$, Vineet Shah ${ }^{1}$, Chitranshu Vashishtha ${ }^{1}$, \\ Sumit Bhatia ${ }^{1}$, Amit Bhasin ${ }^{1}$, Suraj Bhagat ${ }^{1}$, Smruti \\ Ranjan Misra ${ }^{1}$, Randhir Sud ${ }^{1}$
}

\section{ABSTRACT}

Institute of ${ }^{1}$ Digestive and Hepatobiliary Sciences and Department of

${ }^{2}$ Cytopathology, Medanta-The Medicity, Gurgaon, India.

Correspondence: Rajesh Puri

Email:purirajesh69@gmail.com

Methods: We report 10 cases of adrenal histoplasmosis diagnosed by
Background: There have been sporadic reports of histoplasmosis from India. Although traditionally considered a disease of immunocompromised hosts, immunocompetent patients presenting with adrenal histoplasmosis have been reported. Endoscopic ultrasound (EUS) provides easy and safe approach to adrenal glands for fine needle aspiration. endoscopic ultrasound (EUS) guided FNA. The mean age at diagnosis was $54 \pm 9$ years, all were males. Eight patients were immunocompetent while two had cirrhosis; none of them was retrovirus positive. All patients presented with fever and weight loss, 8 had bilateral adrenal enlargement while 2 had isolated left adrenal enlargement. EUS features included hypoechoic $(30 \%)$ or heteroechoic $(70 \%)$ adrenal glands, presence of hypoechoic areas suggestive of necrosis within the gland was identified in $40 \%$ of these patients. Adrenal insufficiency was present in 1 patient $(10 \%)$ which persisted even after successful treatment.

Conclusion: Adrenal histoplasmosis should be considered even in immunocompetent hosts presenting with constitutional symptoms and adrenal enlargement. EUS guided FNA is a safe and accurate modality for adrenal sampling.

KEYWORDS: Adrenal gland; histoplasmosis; immunocompetent; EUS. 


\section{Introduction}

Histoplasmosis is a geographically restricted fungal infection caused by dimorphic fungus Histoplasma capsulatum. ${ }^{1,2}$ Disseminated histoplasmosis is usually seen in immunocompromised hosts and it can involve almost all systems, including the reticuloendothelial system, lungs, gastrointestinal tract, kidneys, central nervous system, bone marrow and adrenal glands. Adrenal glands can be the only site of demonstrable disease in some patients. Early diagnosis and treatment can prevent adrenal insufficiency and its consequences in these patients. Also, accurate diagnosis precludes empirical antittubercular therapy (ATT) in patients with pyrexia of unknown origin.

Ultrasound or computed tomography (CT) guided FNA is commonly used for tissue diagnosis of adrenal enlargement. However, adrenal glands are sometimes difficult to approach by these methods due to their anatomic location. These procedures also have a higher rate of complications and lower accuracy. ${ }^{3}$ Endoscopic ultrasound (EUS) overcomes these limitations due to proximity to target structures (adrenals), is safe and easy to perform in the experienced hands and has good diagnostic accuracy. ${ }^{4-11}$ In this paper, we report ten cases of adrenal histoplasmosis diagnosed by EUS-guided FNA.

\section{Material and Methods}

The study was conducted at a tertiary care high volume EUS centre $(>1500$ EUS examinations per year) in North India. The data was prospectively collected between December 2012 and April 2016. A total of 45 adrenal FNAs were done in the defined period and out of these, 10 were diagnosed as histoplasmosis. All these patients had pyrexia of unknown origin, weight loss and imaging was suggestive of adrenal enlargement without significant lymphadenopathy. The patients were referred from department of internal medicine or endocrinology. All the procedures were carried out in conscious sedation (Midazolam). EUS guided FNA was done using GF-UCT140 linear echoendoscope (EUS scope, Olympus, Tokyo, Japan). The data retrieved from medical records included age, gender, predisposing risk factors, pre-procedure adrenal function, echo-features of adrenal gland, size at short and long axis, type of needle used $(19 \mathrm{G} / 22 \mathrm{G} / 25 \mathrm{G})$, result of FNA and procedural complications.

\section{EUS FNA procedure}

A linear array echoendoscope was inserted into the esophagus and abdominal examination was done to look for porta, liver, left and right adrenal glands and spleen. EUS FNA needle with stylet was introduced in working channel. The left adrenal gland was identified at the level of celiac trunk by rotating the echoendoscope clockwise; or by turning the scope anticlockwise from spleen and identifying left adrenal just above left kidney. Right adrenal gland was imaged in between the upper pole of the right kidney and inferior vena cava from duodenum with the echoendoscope in long position. Doppler was used to avoid any vascular structures in needle path. The stylet was withdrawn slightly before puncture; it was reintroduced fully after puncture of adrenal gland to displace any material in needle (from gastrointestinal wall). After that stylet was completely withdrawn (except in cases where capillary method was used) and about 15 to 20 to and fro movements of needle was done within the gland with application of fanning. The material inside needle was pushed on slides bit by bit with help of stylet. Aspirated material was smeared onto glass slides, few of slides were air dried and rest were wet fixed in 95\% alcohol in Coplin jars. Slides were sent to cytology lab in appropriately labelled slide boxes with test requisition forms having relevant clinical details. Air-dried smears were stained with Giemsa stain after 10 to 15 minutes of methanol fixation. One or two slides were left unstained for any special stains. The fungal morphology was delineated with Periodic Acid Schiff (PAS) and Gomori Groccot Methanamine Silver stains. Alcohol fixed slides were stained with Papanicolou stain. Gomori methenamine silver (GMS) stain was done to confirm histoplasmosis. The type of needle and method of FNA (suction, no suction or capillary) and number of needle passes were operator dependent as we do not have on site cytopathologist facility. 


\section{Results}

A total of 45 adrenal FNA were done in the defined period and out of these, 10 were diagnosed as having histoplasmosis, details of patients are provided in Table 1. The mean age of study group was $54 \pm 9$ years. All patients were immunocompetent males of Indian origin except for two patients who had decompensated cirrhosis (Child B:1; Child C:1). All presented with constitutional symptoms, mainly fever (100\%) for 2 months (median), weight loss $(100 \%)$ and chronic fatigue (100\%). Contrast enhanced abdominal CT was available in all patients which revealed bilateral adrenal enlargement in eight patients $(80 \%)$ and unilateral left adrenal enlargement in two patients. The large axis diameter of larger adrenal gland ranged from 3.0 to $7.6 \mathrm{~cm}$ and short axis ranged from 1.1 to $3.5 \mathrm{~cm}$ in size.

Nine patients underwent EUS-guided FNA of left adrenal gland. Right adrenal was sampled in one patient as the left adrenal was not accessible due to intervening vessels. The needles used were $22 \mathrm{G}$ needle $(\mathrm{n}=4), 25$ $\mathrm{G}$ needle ( $\mathrm{n}=2$, both had decompensated cirrhosis) and 19 G needle $(n=4)$. Median number of passes were 2 (range 1-3). Uniform enlargement of adrenal was noted in all cases. Adrenal gland appeared as hypoechoic $(n=3$, $30 \%)$ or heteroechoic $(n=7,70 \%)$ structure, usually with well-defined borders ( $\mathrm{n}=8,80 \%)$ (Figure 1). The loss of "seagull" shape of adrenal gland was noted in 2 patients (20\%). EUS revealed internal more hypoechoic areas suggestive of necrosis in 4 patients (40\%). EUS guided aspirate was purulent in 3 cases (30\%). In addition, EUS identified presence of small abdominal and/or mediastinal

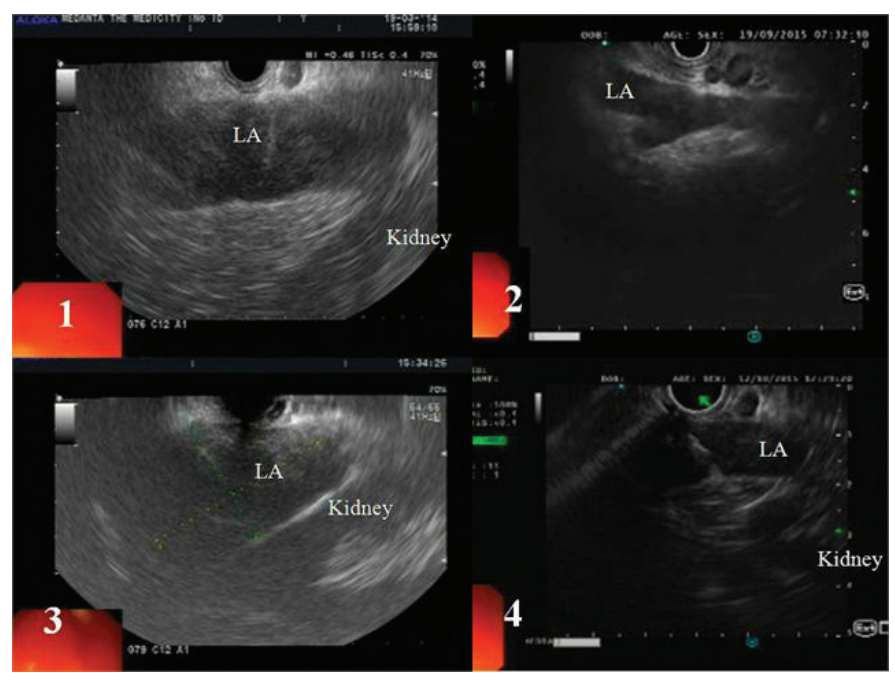

Figure 1: Representative EUS images of left adrenal (LA) glands in 4 patients.

lymphadenopathy in 7 of these patients (70\%), FNA of lymph node was done in one of these cases which revealed chronic granulomatous inflammation.

The cytopathological examination of EUS guided FNA smears revealed inflammatory changes in a necrotic background in all cases (100\%), with presence of granulomas in 2 patients. Capsulated uninucleate intracellular and extracellular yeast forms with morphology consistent with $H$. capsulatum were identified in all. These yeasts stained positive for Periodic Acid Schiff (PAS) and Gomori Groccot Methanamine Silver stains (Figure 2,3).

The patients were treated with Amphotericin B and/or Itraconazole. One patient had adrenal insufficiency and he also received corticosteroids, however, his adrenal

\section{Table 1: EUS features of adrenal glands in 10 patients}

\begin{tabular}{|c|c|c|c|c|c|c|c|c|c|c|}
\hline EUS Features & Case 1 & Case 2 & Case 3 & Case 4 & Case 5 & Case 6 & Case 7 & Case 8 & Case 9 & Case 10 \\
\hline $\begin{array}{l}\text { Adrenal } \\
\text { Enlargement }\end{array}$ & Bilateral & Bilateral & Bilateral & Bilateral & Bilateral & Bilateral & Unilateral & Unilateral & Bilateral & Bilateral \\
\hline Long axis $(\mathrm{cm})$ & 4.2 & 3.0 & 7.6 & 6.0 & 4.3 & 5.7 & 4 & 5.1 & 4.1 & 5.1 \\
\hline Short axis $(\mathrm{cm})$ & 2.0 & 1.1 & 3.2 & 3.0 & 2.5 & 2.8 & 1.5 & 3.5 & 2.6 & 3.0 \\
\hline Echotexture & Hypoechoic & Hypoechoic & Heteroechoic & Heteroechoic & Heteroechoic & Heteroechoic & Heteroechoic & Heteroechoic & Heteroechoic & Hypoechoic \\
\hline Adrenal outline & Ill defined & Well defined & Well defined & Well defined & Well defined & Well defined & Ill defined & Well defined & Well defined & Well defined \\
\hline $\begin{array}{l}\text { Hypoechoic } \\
\text { areas } \\
\text { suggestive of } \\
\text { necrosis }\end{array}$ & No & Yes & Yes & Yes & No & No & Yes & No & No & No \\
\hline
\end{tabular}




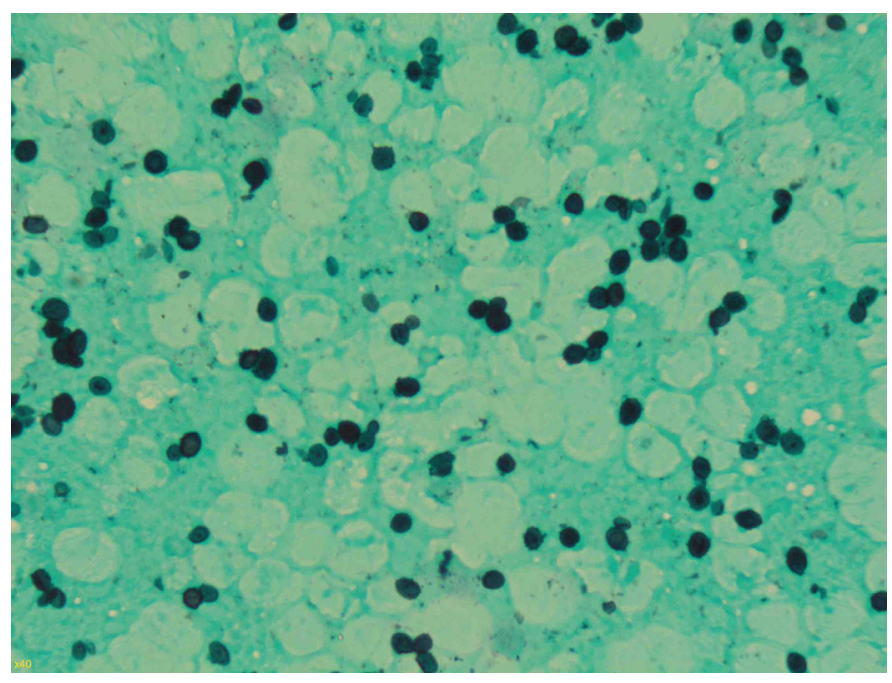

Figure 2: Budding yeasts of Histoplasma capsulatum, stained positive with Methenamine Silver stain.

insufficiency did not improve even after successful treatment. Treatment with antifungals resulted in improvement in all patients.

\section{Discussion}

Histoplasma capsulatum is an intracellular dimorphic fungus that exists in mycelial and budding yeast forms. Although India being a nonendemic area for histoplasmosis, there have been few reports in the recent past, mainly from the eastern parts of the country. ${ }^{12}$ The spores of $\mathrm{H}$. capsulatum is commonly found in soils contaminated with bird droppings. The fungal spores were isolated from Gangetic delta soil. ${ }^{13}$ Still the disease remains under reported owing to its asymptomatic selflimiting course, tuberculosis masquerading the disease and lack of diagnostic facilities.

The spectrum of histoplasmosis ranges from asymptomatic self-limiting disease to progressive disseminated disease involving multiple organs. Disseminated disease is uncommon but frequently involves the adrenal glands (80\%). ${ }^{14}$ Adrenal involvement usually occurs with hematologic dissemination. 30-50\% of patients may have asymptomatic adrenal involvement. ${ }^{15}$ Clinical manifestations include chronic fatigue, weight loss, anorexia and fever of variable duration. ${ }^{16-18}$ However, adrenal insufficiency is uncommon. ${ }^{19}$ We also encountered

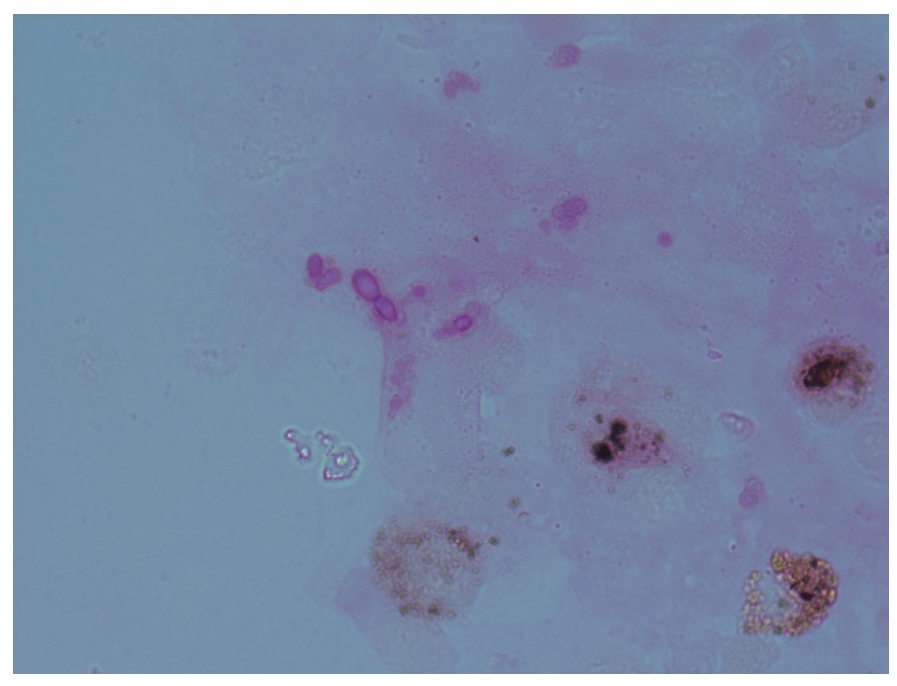

Figure 3: Budding yeasts of Histoplasma capsulatum, stained positive with Periodic Acid Schiff (PAS) stain.

adrenal insufficiency in one among ten patients with adrenal histoplasmosis.

The characteristic CT findings in adrenal histoplasmosis include symmetric adrenal enlargement with preservation of outline and peripheral enhancement with central hypodensity. ${ }^{20}$ Differential diagnosis of bilaterally enlarged adrenal glands include neoplastic masses(malignant and benign), infections liketuberculosis, histoplasmosis, cryptococcosi, blastomycosis, penicillosis, parasitic cysts, trauma, adrenal hemorrhage and autoimmune disease. ${ }^{21-22}$ Ultrasound, CT or EUSguided FNAC can be used for histological diagnosis.

Histoplasmosis should be kept in mind in all necrotising and/or granulomatous lesions of adrenals, more so in immunosuppressed patients. On routine Romanowsky stains ( e.g. Giemsa/MGG/Leishman's), Histoplasma may be discernible as tiny (2 to 5 microns) yeast like dots with clear haloes, often engulfed by histiocytes. But it is hard to distinguish from other yeast like fungi like Cryptococci, hence GMS or PAS stain is imperative.

EUS has evolved as a safe and accurate alternative to ultrasound and CT-guided approaches for obtaining adrenal tissue for histopathological examination. Transabdominal ultrasound can accurately identify left adrenal gland in only $70 \%$ of cases. ${ }^{23}$ Ultrasound and CT-guided approach entails a high risk of 
complications (2.8-8.4\%), including pneumothorax, pain, perinephric hemorrhage, adrenal hematoma and needletract metastasis. ${ }^{3}$ Although EUS-guided FNAC of right adrenal gland is difficult due to its deep anatomic location and proximity to inferior vena cava, left adrenal gland can be easily identified in almost all cases. It appears as a "seagull" shaped structure at the level of celiac trunk. Complications are rare with EUS-guided sampling as it allows real time monitoring of needle passage, thus avoiding nearby organs and blood vessels. Our study suggests that a significant proportion of patients with adrenal enlargement without significant lymphadenopathy may have histoplasmosis and empirical ATT should not be given to such patients unless tissue diagnosis is obtained. Pyrexia of unknown origin is a common entity in south Asian region and practice of empirical antitubercular therapy may result in hepatotoxicity or acute liver failure. ${ }^{24,25}$

In conclusion, adrenal histoplasmosis is not rare in immunocompetent hosts. It should be considered in any patient with adrenal enlargement along with constitutional symptoms. We present the largest series of adrenal histoplasmosis diagnosed by EUS guided FNA. EUS provides a safe and accurate diagnostic modality for adrenal sampling.

\section{References}

1. Adderson E. Histoplasmosis. The Pediatric Infectious. Disease Journal. 25:73-74,2006.

2. Wheat LJ. Histoplasmosis: a review for clinicians from non-endemic areas. Mycoses. 49:274-282,2006.

3. Mody MK, Kazerooni EA, Korobkin M. Percutaneous CTguided biopsy of adrenal masses: immediate and delayed complications. J Comput Assist Tomogr. 1995;19:434-9.

4. Eloubeidi MA, Seewald S, Tamhane A, Brand B, Chen VK, Yasuda I, et al. EUS-guided FNA of the left adrenal gland in patients with thoracic or GI malignancies. Gastrointest Endosc. 2004;59:627-33.

5. Ang TL, Chua TS, Fock KM, Tee AK, Teo EK, Mancer K. EUS-FNA of the left adrenal gland is safe and useful. Ann Acad Med Singapore. 2007;36(11):954-7.

6. DeWitt JM. Endoscopic ultrasound-guided fine-needle aspiration of right adrenal masses: report of 2 cases. J Ultrasound Med. 2008;27(2):261-7.
7. DeWitt J, Alsatie M, LeBlanc J, McHenry L, Sherman S. Endoscopic ultrasound-guided fine-needle aspiration of left adrenal gland masses. Endoscopy. 2007;39(1):65-71.

8. Puri R, Thandassery RB, Choudhary NS, Kotecha H, Misra SR, Bhagat S, et al. Endoscopic ultrasound-guided fine-needle aspiration of the adrenal glands: analysis of 21 patients. Clin Endosc. 2015;48(2):165-70.

9. Azhar JK, Jacqueline HS, Tony LK, Tan BH, Steven JM. Bilateral adrenal histoplasmosis: endoscopic ultrasoundguided fine needle aspiration as a method of diagnosis and assessment. Med J Malaysia. 2011;66(5):504-6.

10. Eloubeidi MA, Luz LP, Crowe DR, Snowden C, Morgan DE, Arnoletti PJ. Bilateral adrenal gland enlargement secondary to histoplasmosis mimicking adrenal metastases: diagnosis with EUS-guided FNA. Diagn Cytopathol. 2010;38(5):357-9.

11. Choudhary NS, Puri R, Kotecha H, Duggal R, Mishra SK, Saraf N. Histoplasmosis Diagnosed by EUS Guided Adrenal FNAC in a Patient with Pyrexia of Unknown Origin. Trop Gastroenterol. 2014;35(4):258-60.

12. Goswami R, Pramanik N, Banerjee D, Raza M, Guha S, Maiti P. Histoplasmosis in eastern India: the tip of the iceberg? Transactions of the Royal Society of Tropical Medicine and Hygiene. 1999;93:540-542.

13. Sanyal M, Thammayya A. Histoplasma capsulatum in the soil of Gangetic plain of India. Indian Journal of Medical Research. 1975;63:1020-1028.

14. Goodwin RA Jr., Shapiro JL, Thurman GH, Thurman SS, Des Prez RM. Disseminated histoplasmosis: clinical and pathologic correlations. Medicine. 1980;59:1-33.

15. Kumar N, Singh S, Govil S. Adrenal histoplasmosis: clinical presentation and imaging features in nine cases. Abdominal Imaging. 2003;28:703-708.

16. Roubsanthisuk W, Sriussadaporn S, Vawesorn N, Parichatikanond P, Phoojaroenchanachai M, Homsanit M, et al. Primary adrenal insufficiency caused by disseminated histoplasmosis: report of two cases. Endocr Pract. 2002;8:237-41.

17. Rozenblit AM, Kim A, Tuvia J, Wenig BM. Adrenal histoplasmosis manifested as Addison's disease: unusual CT features with magnetic resonance imaging correlation. Clin Radiol. 2001;56:682-4.

18. Benevides CF, Durães RO, Aquino B, Schiavon Lde L, Narciso-Schiavon JL, Buzzoleti Fda C. Bilateral adrenal histoplasmosis in an immunocompetent man. Rev Soc Bras Med Trop. 2007;40:230-3.

19. Kauffman CA. Fungal infections in older adults. Clin 
Infect Dis. 2001;33:550-555.

20. Jagat M, Michael V, Lenny T, Lee K. bilateral Adrenal Masses due to Histoplasmosis. J Clin Endocrinol Metab. 2005;90:6725-6726.

21. Gupta P, Bhalla A, Sharma R. Bilateral adrenal lesions. J Med Imaging Radiat Oncol. 2012;56:636-645.

22. Larbcharoensub N, Boonsakan $\mathrm{P}$, Aroonroch R, Rochanawutanon M, Nitiyanant $\mathrm{P}$, Phongkitkarun S, et al. Adrenal histoplasmosis: a case series and review of the literature. Southeast Asian J Trop Med Public Health. 2011;42:920-5.
23. Dietrich CF, Wehrmann T, Hoffmann C, Herrmann G, Caspary WF, Seifert H. Detection of the adrenal glands by endoscopic or transabdominal ultrasound. Endoscopy. 1997;29:859-64.

24. Mir T, Dhobi GN, Koul AN, Saleh T. Clinical profile of classical Fever of unknown origin (FUO). Caspian J Intern Med. 2014;5:35-39.

25. Kumar R, Shalimar, Bhatia V, Khanal S, Sreenivas V, Gupta SD et al. Antituberculosis therapy-induced acute liver failure: magnitude, profile, prognosis, and predictors of outcome. Hepatology. 2010;51:1665-74. 